\title{
Gérer la lombalgie autrement
}

\author{
J.-Y. Cornu
}

(C) Lavoisier SAS 2016

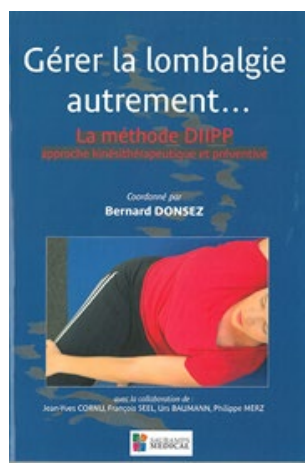

Ce livre collectif est présenté comme une proposition de méthode ; il s'agit de transférer au patient une technique «autoapplicable» pour se «libérer» des contractures qui le gênent. La partie « gestuelle » technique est donc présentée en images dans un chapitre conçu comme un didacticiel, mais en confiant au professionnel le soin de jouer l'intermédiaire pour l'apprendre au patient. Mais ce n'est pas une éducation thérapeutique ni une école du dos, et cette technique n'exclut aucun autre soin ni aucune stratégie à moyen et long termes. La méthode comme la technique découlent de faits d'observation, avant un façonnage sur plus de trois décennies d'exercice professionnel. Ce n'est pas un hasard si ce livre est rédigé par un collectif ayant en commun de recevoir depuis une à quatre décennies des patients lombalgiques ; leur propos est donc d'éviter le passage à la chronicité. Leurs origines professionnelles différentes et surtout leurs exercices transfrontaliers (France, Suisse, Allemagne, Autriche) s'expriment dans la variété des chapitres où se mêlent examen visuel et manuel orthopédique approfondi, utilisation d'outils d'analyse, expérience d'études de cas et enfin réflexion intellectuelle sur les indications intéressantes de cette méthode. La lombalgie est ici replacée dans une approche posturale globale, prenant en compte leurs causes à distance, expliquant l'intérêt que peuvent y trouver des praticiens prenant en charge les problèmes podologiques. Pas une révolution, mais quelque chose de simple et sans danger : à essayer ! 